\title{
Multi-environment QTL studies suggest a role for cysteine-rich protein kinase genes in quantitative resistance to blackleg disease in Brassica napus
}

Nicholas J. Larkan 1,2, Harsh Raman³, Derek J. Lydiate1, Stephen J. Robinson', Fengqun Yu', Denise M. Barbulescu', Rosy Raman ${ }^{3}$, David J. Luckett ${ }^{3}$, Wayne Burton ${ }^{4,5}$, Neil Wratten ${ }^{3}$, Philip A. Salisbury ${ }^{6,7}$, S. Roger Rimmer ${ }^{1 \wedge}$ and M. Hossein Borhan ${ }^{1 *}$

\begin{abstract}
Background: Resistance to the blackleg disease of Brassica napus (canola/oilseed rape), caused by the hemibiotrophic fungal pathogen Leptosphaeria maculans, is determined by both race-specific resistance $(R)$ genes and quantitative resistance loci (QTL), or adult-plant resistance (APR). While the introgression of $R$ genes into breeding material is relatively simple, QTL are often detected sporadically, making them harder to capture in breeding programs. For the effective deployment of APR in crop varieties, resistance QTL need to have a reliable influence on phenotype in multiple environments and be well defined genetically to enable marker-assisted selection (MAS).

Results: Doubled-haploid populations produced from the susceptible B. napus variety Topas and APR varieties AGCastle and AV-Sapphire were analysed for resistance to blackleg in two locations over 3 and 4 years, respectively. Three stable QTL were detected in each population, with two loci appearing to be common to both APR varieties. Physical delineation of three QTL regions was sufficient to identify candidate defense-related genes, including a cluster of cysteine-rich receptor-like kinases contained within a 49 gene QTL interval on chromosome A01. Individual L. maculans isolates were used to define the physical intervals for the race-specific $R$ genes $R / m 3$ and $R / m 4$ and to identify QTL common to both field studies and the cotyledon resistance response.

Conclusion: Through multi-environment QTL analysis we have identified and delineated four significant and stable QTL suitable for MAS of quantitative blackleg resistance in B. napus, and identified candidate genes which potentially play a role in quantitative defense responses to $L$. maculans.

Keywords: Brassica napus, Leptosphaeria maculans, Blackleg, Quantitative resistance, Chitin, CRK

Abbreviations: APR, Adult plant resistance; BLAT, BLAST-like alignment tool; CRK, Cysteine-rich receptor kinase; DH, Doubled haploid; eLRR, Extracellular leucine-rich repeat; ETI, Effector-triggered immunity; HR, Hypersensitive response; ICIM, Inclusive composite interval mapping; II, Internal infection; LG, Linkage group; LOD, Logarithm of the odds; MAS, Marker-assisted selection; MET, Multi-environment trait; NIL, Near-isogenic line; PAMP, Pathogen-associated molecular patterns; PTI, PAMP triggered immunity; QTL, Quantitative trait loci; S, Survival; SA, Salicylic acid; SNP, Single nucleotide polymorphism; SSR, Simple sequence repeat; TC, Topas/AG-castle; TS, Topas/AV-capphire
\end{abstract}

\footnotetext{
* Correspondence: Hossein.Borhan@agr.gc.ca

'Deceased

'Saskatoon Research Centre, Agriculture and Agri-Food Canada, Saskatoon,

SK, S7N 0X2, Canada

Full list of author information is available at the end of the article
}

\section{$\int$ Biomed Central}

(c) 2016 The Author(s). Open Access This article is distributed under the terms of the Creative Commons Attribution 4.0 International License (http://creativecommons.org/licenses/by/4.0/), which permits unrestricted use, distribution, and reproduction in any medium, provided you give appropriate credit to the original author(s) and the source, provide a link to the Creative Commons license, and indicate if changes were made. The Creative Commons Public Domain Dedication waiver (http://creativecommons.org/publicdomain/zero/1.0/) applies to the data made available in this article, unless otherwise stated. 


\section{Background}

Prevention of catastrophic crop loss to plant pathogens is most often achieved through the incorporation of resistance genetics into commercial cultivars. Host responses to plant pathogens are broadly divided into two categories; basal defense responses induced by generic pathogen signals or elicitors called "pathogen-associated molecular patterns" (PAMPs), resulting in mild defense responses collectively known as 'PAMP triggered immunity' (PTI) and $R$ gene mediated 'effector triggered immunity' (ETI) in which race-specific pathogen avirulence (Avr) proteins trigger robust defense mechanisms including hypersensitive response (HR) leading to host cell death at the site of infection [1]. When studying foliar plant pathogens, the HR response of race-specific $R$ genes often provides a visual phenotype, indicating an incompatible interaction and allowing for the determination of pathogen virulence. This distinction is used to separate specific $R$ gene interactions from quantitative resistance which can provide effective 'adult plant resistance' (APR) within a crop variety through the cumulative action of multiple resistance loci. APR is usually measured at the end of the growing season in field trials.

APR is particularly important for combating diseases of Brassica napus L. (canola/oilseed rape) in which $R$ gene mediated resistance is lacking, such as Sclerotinia Stem Rot (Sclerotinia sclerotiorum) [2-4] and Verticillium Wilt (Verticillium longisporum) [5-7] or for diseases where pathogen populations often display a rapid adaptation towards $R$ gene mediated resistance, such as in the case of blackleg disease, caused by the hemibiotrophic fungal pathogen Leptosphaeria maculans $[8,9]$.

Avoidance of $R$ gene mediated resistance by $L$. maculans can occur both rapidly and in a geographically localised fashion when a pathogen population is under heavy selection pressure. A rapid decline in the efficiency of the blackleg $R$ gene $R \operatorname{lm} 1$ in controlling the disease in Europe highlighted the evolutionary potential of the pathogen [10]. A high frequency of mutation and deletion of the L. maculans avirulence gene AvrLm4-7 was reported to occur within a small plot area sown continually to $B$. napus harbouring $R \operatorname{lm} 7$, while virulent pathotypes remained undetectable in samples from the surrounding local pathogen population [11]. High rates of infection were observed in some areas of Australia in canola varieties carrying the $R$ gene LepR3 only three years after first commercial release of the material [12], though this rapid loss of effective resistance may have been aided by pre-exposure to $R \operatorname{lm} 1$ varieties, as avirulence towards $L e p R 3$ and $R \operatorname{lm} 1$ is conferred by the same L. maculans avirulence gene; AvrLm1 [13].

$B$. napus cultivars containing only APR usually show no difference in the development of leaf lesions when compared with susceptible cultivars, yet they restrict the development of internal stem infection by the pathogen, resulting in lower levels of crown canker formation [14]. This is in contrast to $R$ gene mediated resistance which leads to arrest of $L$. maculans growth at the site of infection on cotyledons and leaves. When major $R$ gene mediated resistance is avoided by virulent strains within the mixed pathogen population, APR reduces the selection and proliferation of virulent pathotypes in crop residues and the potential for catastrophic crop loss in following seasons [15-17].

While $R$ gene mediated resistance can often be detected efficiently and rapidly by observing hypersensitive response after inoculation of $B$. napus cotyledons with well-characterised L. maculans isolates, assessment of APR is much more difficult. Resistance needs to be measured either through field-based studies, or under controlled conditions through infection with single spore-derived L. maculans isolates and assessment of stem infection in plants grown for several months $[18,19]$. Assessment of APR in field-based studies can be difficult considering the complexity of plant-pathogenenvironment interactions. Populations of L. maculans in most disease nurseries are genetically heterogeneous mixtures arising from sexual recombination and variation of pathotypes should be expected both within a trial site and between trial years. Also, variation of host response due to heterozygosity of $B$. napus lines may be confused for polygenic control of resistance [20]. There has also been a widely-held view that blackleg APR is race non-specific [17], based largely on experience of the French variety Jet Neuf, which provided durable resistance to blackleg disease over many years in Europe and was also utilised in early efforts to improve blackleg resistance in Australian germplasm [21, 22]. However, more recent studies utilising single $L$. maculans isolates have questioned the "race non-specific" nature of blackleg APR $[19,23]$.

Maintenance of strong APR in canola varieties can most efficiently be achieved through marker-assisted breeding based on the molecular characterisation of quantitative trait loci (QTL) associated with resistance [17]. The French variety Darmor, derived from Jet Neuf, is the most extensively studied $B$. napus variety harbouring quantitative resistance to L. maculans. A doubled-haploid (DH) population produced from a cross between Darmor- $b z h$ and the susceptible Korean cultivar Yudal (DY) was utilised to map 10 QTL contributing to blackleg resistance, with four of the QTL detected stably across two years of field testing [24]. The resistance was further analysed in Darmor x Samouraï (DS) DH and $\mathrm{F}_{2}$ populations, revealing four QTL that were common to both the DY and DS populations [25]. Near-isogenic lines (NILs) were also produced for four Darmor QTL; LmA2, LmA9, LmC2 and $L m C 4$, though only $L m A 2$ was fully validated as having a significant effect on reducing disease severity [26]. 
Blackleg APR has also been assessed in several Australian varieties, revealing multiple QTL that are potentially common to several Australian and French cultivars $[9,19,27]$.

Little is known about the molecular basis of APR to $L$. maculans infection in Brassica species. While two racespecific genes responsible for ETI-mediated blackleg resistance, LepR3 and Rlm2, have been cloned from $B$. napus and shown to encode extracellular leucine-rich repeat (eLRR) receptor-like proteins recognising the $L$. maculans effectors AvrLm1 and AvrLm2, respectively $[13,28,29]$, no genes underpinning blackleg resistance QTL have been identified. Infection of B. napus by $L$. maculans results in attempted physical restriction of the pathogen by the host, via callose deposition, while an increased lignification response has also been reported for APR varieties [30, 31]. L. maculans infection triggers induction of the salicylic acid (SA) signalling pathway [31, 32] which plays a critical role in plant defense [33]. SA signalling can be triggered in B. napus by purified $L$. maculans cell wall components [34] and is greatly induced during ETI, along with the ethylene signalling pathway and $\mathrm{H}_{2} \mathrm{O}_{2}$ accumulation [31, 32, 35]. However, these studies have all focused on early infection events in the cotyledons of $B$. napus seedlings; nothing is known about which defense mechanisms may be active against the invading hyphae as they grow asymptomatically through the petiole [18] and stem [36].

In this study we identified several stable blackleg resistance QTL, with resistance alleles derived from AG-Castle and AV-Sapphire, two blackleg-resistant Australian $B$. napus varieties released in 2002 and 2003, respectively. We used Topas/AG-Castle (TC) and Topas/AV-Sapphire (TS) DH populations to assess the APR of the varieties over multiple years at two locations, performed both single- and multi-environment QTL mapping and defined the physical locations of the QTL relative to the recently released B. napus Darmor-bzh reference genome [37], allowing for the identification of candidate defense-related genes.

\section{Results \\ Population data}

Field tests were conducted in south eastern Australia in disease nurseries located near Horsham, Victoria and Wagga Wagga, New South Wales (Fig. 1). Mean survival percentages (S) ranged from 25.9 to $43.2 \%$ for the TC population, and 11.2 to $69.2 \%$ for the TS population, with $\mathrm{S}$ of individual entries (3 to 4 entries per DH line) ranging from 0 to $100 \%$ in all tests except for TS Horsham 2008, where the maximum $S$ recorded for a single entry was $71.1 \%$. Mean internal infection percentages (II) ranged from 38.5 to $58.6 \%$ for the TC population and 45 to $87.4 \%$ for the TS population. The minimum II observed was $4 \%$ (TC Wagga Wagga 2010 and TS Wagga Wagga 2009) with a maximum II of $100 \%$ recorded in all tests. For both populations, mean survival was always higher, and mean internal infection was always lower, in tests at the Wagga Wagga site when compared to the Horsham site (Table 1, Additional file 1: Figure S1). Heritability was calculated based on total entries in each environment for each scoring metric; survival (S) and internal infection (II) and was generally high, producing similar ranges for each metric (S: $0.75-0.9$, II: 0.73-0.89) (Table 1).

\section{Linkage analysis}

While the lack of heterozygosity in the populations showed the lines produced to be true doubled haploids,

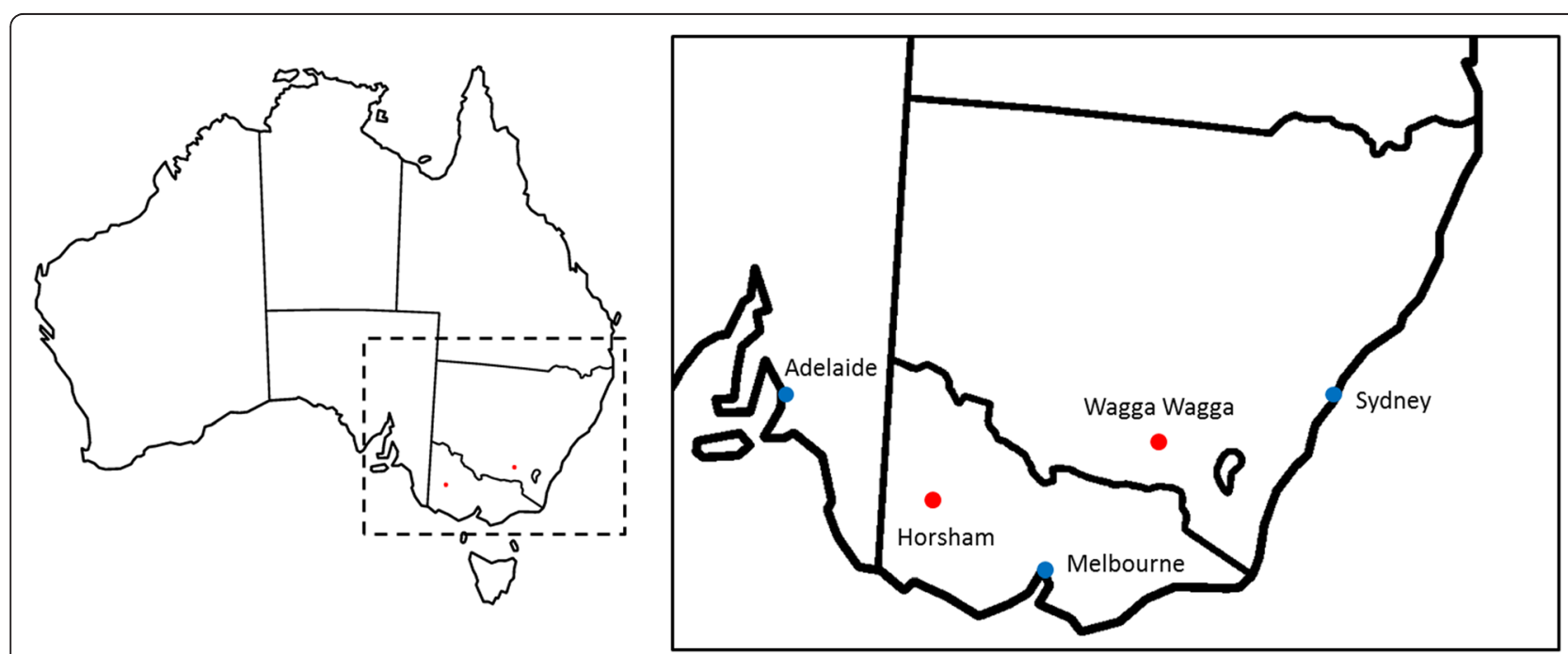

Fig. 1 Location of field trial sites in south eastern Australian. Dashed box on left indicates highlighted region on right. Red dots show location of trial sites, blue dots show major cities. Map modified from original image (https://commons.wikimedia.org) 
Table 1 Survival, internal infection and heritability of DH populations in two environments

\begin{tabular}{|c|c|c|c|c|c|c|c|c|c|c|}
\hline Population & Location & Year & Blocks & Metric & Range (\%) & Mean (\%) & Med.(\%) & $\sigma_{A}^{2}$ & $\sigma_{E}^{2}$ & $h^{2}$ \\
\hline Topas/ & Horsham & 2009 & 3 & $S$ & $0-100$ & 25.9 & 20 & 1199.6 & 148.7 & 0.89 \\
\hline \multirow[t]{5}{*}{ AG-Castle } & & & & $\|$ & $12-100$ & 58.6 & 56 & 1616.2 & 277.4 & 0.85 \\
\hline & Wagga Wagga & 2009 & 3 & S & $0-100$ & 43.2 & 43 & 590.5 & 197 & 0.75 \\
\hline & & & & $\|$ & $12-100$ & 56.2 & 56 & 729.5 & 193 & 0.79 \\
\hline & Wagga Wagga & 2010 & 4 & S & $0-100$ & 38.6 & 38 & 1106.4 & 165.9 & 0.87 \\
\hline & & & & $\|$ & $4-100$ & 38.5 & 36 & 741.8 & 120.7 & 0.86 \\
\hline Topas/ & Horsham & 2008 & 3 & S & $0-71.1$ & 11.2 & 6.8 & 419 & 44.5 & 0.90 \\
\hline \multirow[t]{7}{*}{ AV-Sapphire } & & & & ॥ & $8-100$ & 62.9 & 64 & 1937 & 263 & 0.88 \\
\hline & Wagga Wagga & 2009 & 3 & S & $0-100$ & 32 & 28 & 1069 & 223.4 & 0.83 \\
\hline & & & & $\|$ & $4-100$ & 45.4 & 40 & 941.4 & 348.7 & 0.73 \\
\hline & Wagga Wagga & 2011 & 4 & S & $0-100$ & 69.2 & 72.8 & 1325.2 & 205.1 & 0.87 \\
\hline & & & & $\|$ & $16-100$ & 45 & 44 & 632.2 & 123.4 & 0.84 \\
\hline & Horsham & 2012 & 4 & S & $0-100$ & 16.5 & 9 & 1051.8 & 155.7 & 0.87 \\
\hline & & & & $\|$ & $24.2-100$ & 87.4 & 94 & 700.4 & 85.1 & 0.89 \\
\hline
\end{tabular}

Data given for each population (Topas/AG-Castle or Topas/AV-Sapphire) in each environment (location $\mathrm{x}$ year)

Blocks represent replicates per trial, scoring metrics; S survival, I/ internal infection

Range, mean and median (Med.) given for total entries (blocks $x \mathrm{DH}$ lines). $\sigma_{\mathrm{A}}^{2}=$ variance (additive), $\sigma_{\mathrm{E}}^{2}=$ variance (environmental); $h^{2}=$ heritability $\left(\sigma_{\mathrm{A}}^{2} / \sigma_{\mathrm{A}}^{2}+\sigma_{\mathrm{E}}^{2}\right.$ )

marker distortion was detected in many regions. $40 \%$ of TC marker bins and $20.8 \%$ of TS markers showed mild distortion (failed to conform to a $1: 1$ ratio; Chi-square test, $p=0.05$ to 0.001 ), while $21 \%$ of TC markers and $6 \%$ of TS markers showed severely distorted segregation $(p<0.001)$. The severely affected regions were generally towards the ends of chromosomes; in the TC population they were the upper portions of chromosomes A02, C01, $\mathrm{C} 03$ and $\mathrm{C} 09$, and the lower portions of A05, C01 and C06. Additionally, the entire A07 chromosome was uniformly distorted, with all markers favoring the Topas parent allele in an approximately 3:1 ratio. In the TS population, upper $\mathrm{C} 03$, lower A08 and all of $\mathrm{C} 02$ were severely distorted. Two draft maps were initially produced for each population; one containing all markers, and a second in which all severely affected marker loci were removed. Whole-genome QTL analysis was performed using both draft map versions. No changes to QTL positions were observed between draft maps, nor were significant differences in QTL LOD and variance scores, with the exception of the Rlm3 locus, positioned on A07 on the 'distorted' TC map and absent from the 'non-distorted' map. To accommodate mapping of the A07 Rlm3 resistance locus and its associated QTL in the final analysis, TC A07 was resolved independently and added to final TC map. All other distorted markers were removed prior to final map construction. The final TC map consisted of 307 marker bins (collections of cosegregating markers) spanning $2182.3 \mathrm{cM}$ in 21 linkage groups (LGs) representing all 19 B. napus chromosomes. Linkage mapping for the TS population produced 23 LGs (199 bins, $1714.97 \mathrm{cM}$ ), which were assigned to 18 of the
19 chromosomes, with no representation for chromosome C02.

\section{Single-environment QTL}

Permutation tests performed for each scoring metric, survival (S) and internal infection (II) percentage, in each environment determined significant LOD thresholds between 2.76 and 3.07 for the TC population and 2.66-2.95 for the TS population. Analysis of the singleenvironment data produced multiple QTL exceeding their respective LOD thresholds (LOD 3.37-41.47), which were localised to seven chromosomes for the TC population (A01, A08, C03, C04, C05, C06 and C07) and five chromosomes of the TS population (A01, A03, A09, C01 and C06), and accounting for between 2.48 and $31.77 \%$ of the phenotypic variance (Additional file 2: Table S1). To identify chromosomal regions harbouring 'stable' and significant QTL regions, QTL identified from individual environments were only considered significant if they exceeded both the LOD threshold for each analysis, based on permutation test (1000 permutations, 0.05 error) and accounted for $>5 \%$ of the variance. After applying these criteria three significant QTL, each with favorable alleles derived from the respective resistance donor parent (AG-Castle or AV-Sapphire), were identified in each population. These regions consisted of clustered QTL located on chromosomes A01, A08 and C06 of AG-Castle, and A01, A09 and C06 of AV-Sapphire, with the A01 and C06 QTL regions appearing to be common to both resistance donor parent lines (Table 2, Fig. 2). For the TC larger population (242 lines), the three clustered QTL regions were represented by QTL 
Table 2 Clustered single and multi-environment QTL detected in TC and TS populations

\begin{tabular}{|c|c|c|c|c|c|c|c|c|c|c|c|}
\hline Trait $^{\mathrm{a}}$ & Chrom. & QTL Int. (cM) & Support interval & Peak (cM) & Peak interval & LOD & $\sigma^{2}(\%)$ & Add & $\sigma_{\mathrm{A}}^{2}$ & $\sigma_{E}^{2}$ & $h^{2}$ \\
\hline \multicolumn{12}{|c|}{ A) Topas/AG-Castle } \\
\hline W09 S & A01 & $54-67.5$ & sR9564 - sN12790 & 59 & sN11665 - sN12790 & 5.20 & 8.32 & 4.61 & & & \\
\hline $\mathrm{H} 09 \mathrm{~S}$ & A01 & $68-69$ & sN12790 - sN4638 & 69 & sR8420 - sN4638 & 17.20 & 15.84 & 7.80 & & & \\
\hline W10 S & A01 & $68-69$ & sN12790 - sN4638 & 69 & sR8420 - sN4638 & 24.25 & 29.90 & 9.31 & & & \\
\hline W10 II & A01 & $68-69$ & sN12790 - sN4638 & 69 & sR8420 - sN4638 & 9.08 & 11.52 & 4.90 & & & \\
\hline MET II & A01 & 69 & sR8420 - sN4638 & 69 & sR8420 - sN4638 & 34.85 & 20.55 & 6.53 & 12.06 & 8.49 & 0.59 \\
\hline MET S & A01 & $68.5-69$ & sN12790 - sN4638 & 69 & sR8420-sN4638 & 41.47 & 15.84 & 5.79 & 10.80 & 5.04 & 0.68 \\
\hline $\mathrm{HO9} \|$ & A01 & $69-69.5$ & sR8420 - sN12176 & 69.5 & sN4638 - sN12176 & 25.71 & 31.77 & 14.01 & & & \\
\hline CotQTL & A07 & $73.5-74$ & sNRA59 - sR12387b & 73.5 & RIm3 - sR12829 & 72.37 & 72.18 & 23.29 & & & \\
\hline $\mathrm{H} 09 \mathrm{~S}$ & A08 & $34.5-42.5$ & sN4513Fa - sNRB88 & 39 & sN4513Fa - sNRG04 & 8.96 & 7.63 & 5.49 & & & \\
\hline W09 S & A08 & $34.5-42.5$ & sN4513Fa - sNRB88 & 39 & sN4513Fa - sNRG04 & 4.45 & 7.05 & 4.32 & & & \\
\hline MET S & A08 & $37.5-42$ & sN4513Fa - sNRB88 & 39 & sN4513Fa - sNRG04 & 13.59 & 5.13 & 3.51 & 3.85 & 1.28 & 0.75 \\
\hline H09 ॥ & A08 & $39.5-47.5$ & sNRG04 - sN12352a & 41 & sNRG04 - sNRB88 & 7.42 & 7.85 & 7.08 & & & \\
\hline MET II & A08 & $39.5-46.5$ & sNRG04 - sN12352a & 41.5 & sNRG04 - sNRB88 & 10.29 & 5.26 & 3.39 & 3.16 & 2.10 & 0.60 \\
\hline W10 S & A08 & $43-50.5$ & sNRB88 - sR9433 & 46 & sNRB88 - sN12352a & 8.34 & 9.08 & 5.19 & & & \\
\hline W09 II & C06b & $21-22$ & brPb - 841625 - brPb - 841355 & 21.5 & brPb - 841625 - brPb - 841355 & 3.04 & 5.17 & 3.50 & & & \\
\hline $\mathrm{H} 09 \mathrm{~S}$ & C06b & $23.5-45$ & brPb - 841355 - sN12461lx & 40 & brPb - 841355 - sN12461lx & 14.59 & 14.08 & 7.42 & & & \\
\hline CotQTL & C06b & $23.5-45$ & brPb - 841355 - sN12461lx & 40 & brPb - 841355 - sN12461lx & 4.71 & 9.26 & 7.25 & & & \\
\hline MET S & C06b & $36.5-45$ & brPb - 841355 - sN12461lx & 42.5 & brPb - 841355 - sN12461lx & 25.99 & 9.22 & 5.06 & 8.12 & 1.10 & 0.88 \\
\hline W10 ॥ & C06b & $23.5-45$ & brPb - 841355 - sN12461lx & 44.5 & brPb - 841355 - sN12461lx & 5.88 & 7.35 & 3.94 & & & \\
\hline H09 ॥ & C06b & $23.5-45$ & brPb - 841355 - sN12461lx & 45 & brPb - 841355 - sN12461lx & 10.44 & 11.07 & 8.34 & & & \\
\hline W10 S & c06b & $23.5-45$ & brPb - 841355 - sN12461lx & 45 & brPb - 841355 - sN12461Ix & 10.00 & 10.83 & 5.65 & & & \\
\hline MET II & C06b & $38-45$ & brPb - 841355 - sN12461lx & 45 & brPb - 841355 - sN12461lx & 16.44 & 7.83 & 4.30 & 5.14 & 2.70 & 0.66 \\
\hline \multicolumn{12}{|c|}{ B) Topas/AV-Sapphire } \\
\hline $\mathrm{H} 12 \mathrm{~S}$ & A01 & $30.5-39$ & sR9228a - sR9555x & 36.5 & sR6202b - sR9555x & 4.3901 & 10.10 & 5.33 & & & \\
\hline MET S & $\mathrm{A} 01$ & $33.5-39$ & sR9228a - sR9555x & 36.5 & sR6202b - sR9555x & 13.1741 & 10.07 & 5.04 & 8.71 & 1.36 & 0.86 \\
\hline W11 ॥ & $\mathrm{A} 01$ & $30.5-39$ & sR9228a - sR9555x & 37.5 & sR6202b - sR9555x & 3.4382 & 11.07 & 4.39 & & & \\
\hline W11 S & A01 & $30.5-39$ & sR9228a - sR9555x & 39 & sR6202b - sR9555x & 6.352 & 18.47 & 8.21 & & & \\
\hline $\mathrm{H} 12$ ॥ & $\mathrm{A} 01$ & $36.5-40$ & sR6202b - sN12176 & 39.5 & sR9555x - sN12176 & 3.3711 & 9.00 & 4.08 & & & \\
\hline MET II & $\mathrm{A} 01$ & $36.5-40$ & sR6202b - sN12176 & 40 & sR9555x - sN12176 & 7.8313 & 5.02 & 4.20 & 4.74 & 0.28 & 0.94 \\
\hline CotQTL & $\mathrm{A} 07$ & $48-56.5$ & sN2555Ra - RIm4 & 56 & sN2555Ra - R/m4 & 30.80 & 48.92 & 19.16 & & & \\
\hline W11 ॥ & A09 & $5-19.5$ & sS2212 - sR9373 & 8 & sR6410 - sR9373 & 5.35 & 16.38 & 5.41 & & & \\
\hline $\mathrm{H} 12 \|$ & A09 & $6.5-14.5$ & sS2212 - sR9373 & 8 & sR6410 - sR9373 & 3.05 & 8.12 & 3.92 & & & \\
\hline W11 S & A09 & $5-19.5$ & sS2212 - sR9373 & 8 & sR6410 - sR9373 & 5.34 & 15.00 & 7.48 & & & \\
\hline MET S & A09 & $6.5-12.5$ & sS2212 - sR9373 & 8 & sR6410 - sR9373 & 10.41 & 9.18 & 4.67 & 7.33 & 1.86 & 0.80 \\
\hline W09 II & A09 & $8-11.5$ & sR6410 - sR9373 & 8.5 & sR6410 - sR9373 & 2.89 & 11.83 & 6.68 & & & \\
\hline MET II & A09 & $8-14$ & sR6410 - sR9373 & 9.5 & sR6410 - sR9373 & 13.97 & 9.66 & 5.88 & 9.10 & 0.56 & 0.94 \\
\hline H08 II & A09 & $21-29$ & sR9373 - sR6966 & 25 & sR9373 - sR6966 & 3.43 & 12.31 & 9.82 & & & \\
\hline $\mathrm{H} 12 \mathrm{~S}$ & A09 & $21.5-33.5$ & sR9373 - sR12078a & 27.5 & sR9373 - sR6966 & 3.41 & 9.47 & 5.19 & & & \\
\hline H08 II & $\mathrm{C06}$ & $38-60$ & sS2486a - sN5088F & 52.5 & sS2486a - sR12387a & 4.79 & 19.31 & 12.24 & & & \\
\hline $\mathrm{H} 12 \mathrm{~S}$ & C06 & $35.5-60$ & sS2486a - sN5088F & 54.5 & sS2486a - sR12387a & 6.91 & 22.18 & 7.88 & & & \\
\hline $\mathrm{H} 12$ ॥ & C06 & $40.5-60$ & sS2486a - sN5088F & 56.5 & sS2486a - sR12387a & 4.55 & 13.63 & 5.00 & & & \\
\hline
\end{tabular}


Table 2 Clustered single and multi-environment QTL detected in TC and TS populations (Continued)

\begin{tabular}{|c|c|c|c|c|c|c|c|c|c|c|c|}
\hline MET II & $\mathrm{C} 06$ & $50.5-58.5$ & sS2486a - sN5088F & 57 & sS2486a - sR12387a & 10.84 & 9.67 & 4.97 & 6.69 & 2.98 & 0.69 \\
\hline H08 S & C06 & $43-60$ & sS2486a - sN5088F & 58 & sS2486a - sR12387a & 4.58 & 15.26 & 4.73 & & & \\
\hline MET S & C06 & $53.5-58.5$ & sS2486a - sN5088F & 58 & sS2486a - sR12387a & 14.65 & 9.34 & 5.04 & 8.76 & 0.58 & 0.94 \\
\hline
\end{tabular}

Significant QTL shown only (Single environment QTL $>5 \sigma^{2}(\%)$, MET QTL $>5 \sigma^{2}(\%)$ and $h^{2}>0.5$ )

${ }^{a}$ Single environment (plain) and multi-environment (bold) QTL, single-environment trait names given as location $(\mathrm{H}=\mathrm{Horsham}, \mathrm{W}=\mathrm{Wagga}$ Wagga), year (08-12 = 2008-2012) and metric ( $\mathrm{S}=$ survival, II = internal infection), MET = Multi-environment traits (all environments) for $S$ (survival) and MET II (internal infection) metrics, CotQTL = single-isolate cotyledon tests. Chrom. = B. napus chromosome; QTL Int. (cM) = QTL interval (in centiMorgans); Support Interval = map interval, defined by flanking markers, which contains QTL (LOD > significance threshold); Peak (cM) = Position of Peak LOD value (in centiMorgans); Peak Interval = map interval containing QTL peak LOD; LOD = peak logarithm of odds; $\sigma^{2}(\%)=$ variance (total percentage); Add = additive effect (positive score indicates net genetic contribution from AG-Castle or AV-Sapphire parent); $\sigma_{\mathrm{A}}^{2}$ variance (additive) portion (\%), $\sigma_{\mathrm{E}}^{2}$ variance (environmental) portion $(\%), h^{2}$ heritability $\left(\sigma_{\mathrm{A}}^{2} / \sigma^{2}(\%)\right)$

detected from all three single-environment analyses (Horsham 2009, Wagga Wagga 2009 \& 2010), accounting for 8.32 to $31.77 \%$ (A01), 7.05 to $9.08 \%$ (A08) and 5.17 to $14.08 \%(\mathrm{C} 06)$ of the phenotypic variance. For the TS population (109 lines), only the A09 QTL region was represented by QTL detected in all four tested environments (Horsham 2008 \& 2012, Wagga Wagga 2009 \& 2010). The TS A01 QTL region was represented by QTL detected in the two 4-block trials (Wagga Wagga 2011, Horsham 2012) which accounted for 9.00 to $18.47 \%$ of the variance in those years. An additional A01 QTL (11.60 \% variance) was also detected from the Wagga Wagga 2009 trial (3-block) though this failed to exceed the LOD threshold for significance. The TS C06 QTL region was only represented by QTL detected in the two Horsham nurseries (2008 \& 2012), though a large portion of the variance was attributed to this region in those tests (13.63 to $22.18 \%$ ).

\section{Multi-environment QTL}

Multi-Environment Trait (MET) analysis performed using S and II data for all environments produced MET QTL corresponding to each of the seven chromosomes previously identified from the single-environment analysis, except for TC A10 and C07. In addition, multiple lowvariance MET QTL were detected for the TS population. Estimates of the additive genetic (A) and environmental (E) components of the multi-environment variance $\left(\sigma^{2}\right)$ were used to calculate the narrow-sense heritability $\left(h^{2}\right)$ of each MET QTL. As doubled-haploid populations were used for the study there was no heterozygosity and thus no dominance component for the variance. $h^{2}$ values between 0.59 and 0.88 were determined for the TC QTL, while slightly higher $h^{2}$ values (0.69-0.94) were determined for QTL in the smaller TS population (Additional file 2: Table S1). Multi-Environment Traits (MET) were considered significant, and the associated chromosomal regions harbouring MET QTL were considered to make a stable contribution to resistance, if they accounted for greater than $5 \%$ of the total variance and were attributed a heritability score of 0.5 or higher. After applying these criteria only MET QTL associated with the previously-identified single-environment QTL clusters were retained. The total multi-environment survival (MET S) and internal infection (MET II) variance explained by the major QTL regions was 30.19 and $33.64 \%$, respectively, for the TC population, and 28.59 and $24.35 \%$, respectively, for the TS population (Table 2).

\section{Single-isolate characterisation}

Each DH line in the TC and TS populations were characterised for the presence or absence of hypersensitive response via cotyledon infection tests, as differential phenotypic reactions were initially observed to the $L$. maculans isolates WA30 (avirulent on AG-Castle, virulent on AV-Sapphire) and v23.1.3 (virulent on AG-Castle, avirulent on AV-Sapphire). After the phenotypic data was converted to Mendelised resistant $(+)$ and susceptible (-) scores and incorporated into the genetic maps for each population, the cotyledon resistance loci were determined to localise to chromosome A07, co-segregating with the simple sequence repeat (SSR) marker sR12173 in both cases (Fig. 2). As chromosome A07 is known to harbour the race-specific blackleg resistance genes $R \operatorname{lm} 1, R \operatorname{lm} 3$, $R \operatorname{lm} 4, R \operatorname{lm} 7$ and $R \operatorname{lm} 9$ [19, 38-40] further characterisation of the parental lines with differential $L$. maculans isolates varying in their reactions to the A07 $R$ genes was performed. Only isolates avirulent towards $R \operatorname{lm} 3$ or $R \operatorname{lm} 4$ produced resistant reactions on AG-Castle or AVSapphire, respectively (Table 3). Additional evidence for the presence of $R \operatorname{lm} 4$ in AV-Sapphire was produced using the transgenic isolate 3R11: AvrLm4-7, which demonstrated the AvrLm4-7 gene conveys avirulence towards AV-Sapphire (Table 3, Additional file 3: Figure S2).

The Rlm3 locus of the TC population co-segregated with a group of three SSR alleles; sR12294a, sR12173 and sR2834a (Fig. 2), which span a region of 80 genes of the $B$. napus Darmor-bzh reference genome [37] on chromosome A07 (BnaA07g20270D to BnaA07g21070D). Unfortunately the closest flanking markers to the Rlm3 cluster were the SSR markers SNRA59 and sR12829, both of which match to portions of the genomic sequence which are not currently incorporated into the B. napus chromosome A07 model (matches to 'chrUn_random' and 'chrA07_random', respectively). The next-closest flanking markers, sR12119a (closest gene $=$ BnaA07g17900D) and 


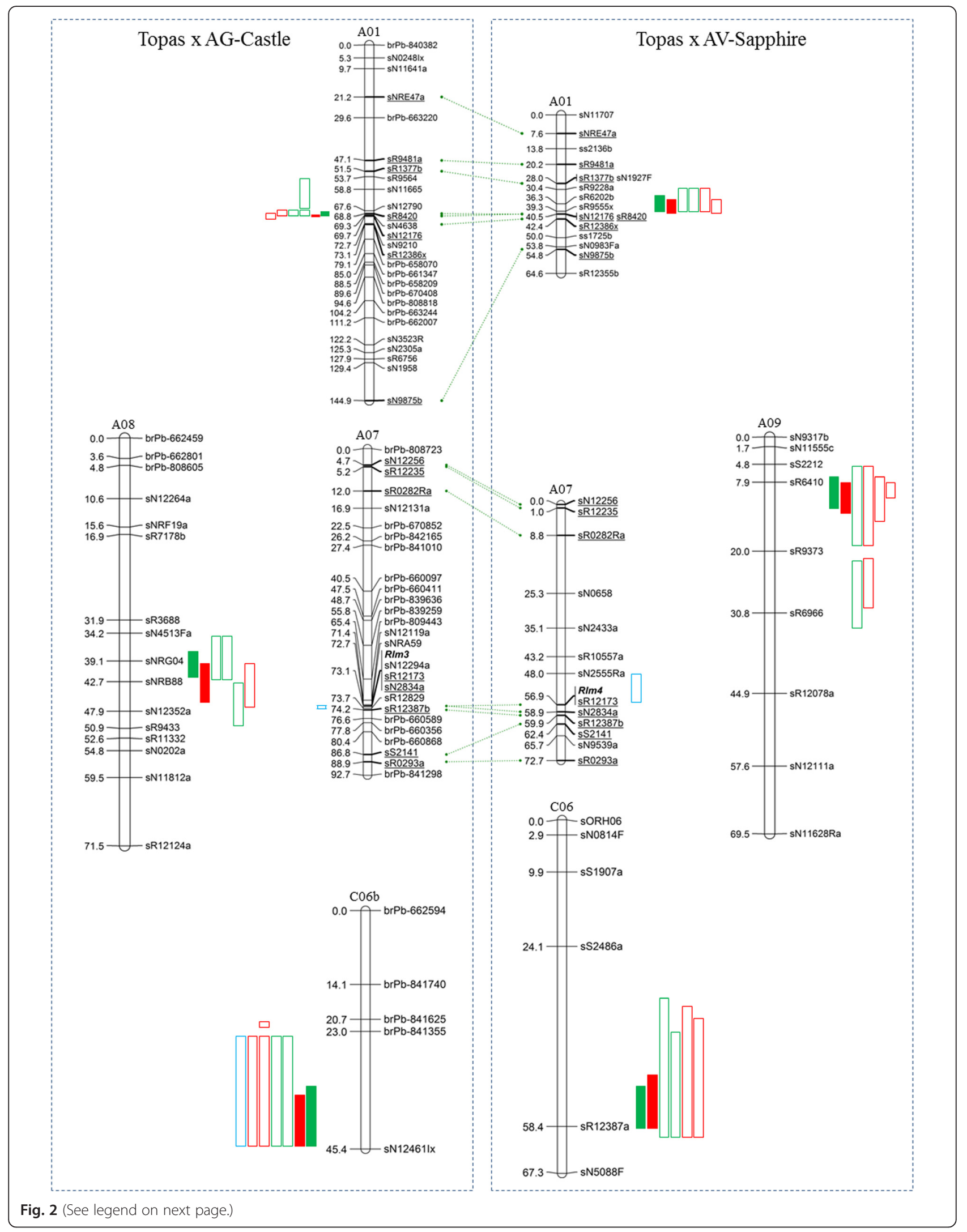


(See figure on previous page.)

Fig. 2 Significant QTL clusters for TC and TS Populations. Linkage maps shown only for B. napus chromosomes harbouring significant QTL (TC: A01, A07, A08 \& C06; TS: A01, A07, A09, C06). QTL for survival (green), internal infection (red) and cotyledon (blue) metrics. Single-environment QTL shown as open boxes, multi-environment QTL as solid boxes. Green dotted lines indicate common markers. Positions of blackleg $R$ genes R/m3 and RIm4 shown in bold

sR12387b (BnaA07g26220D) represent a region spanning approximately $4.5 \mathrm{Mb}$ and 832 genes of $B$. napus chromosome A07. Utilising the $B$. rapa genome sequence [41] we were able to define the smaller sNRA59-sR12829 interval as spanning the genes Bra003406 through Bra004064 (658 genes) on $B$. rapa $\mathrm{A} 07$, and to infer an equivalent $B$. napus A07 interval of not greater than BnaA07g18610D to BnaA07g24890D (3.5 Mb, 628 genes). This suggested that approximately 30 more genes within the $B$. napus interval were not currently incorporated in the current $B$. napus A07 chromosome build [37].
The mapping of $R \operatorname{lm} 4$ with the TS population placed the gene between the SSR markers sN2555Ra and sN2834a, a region spanning approximately $3.9 \mathrm{Mb}$ and 704 genes on chromosome A07 (BnaA07g14030D-BnaA07g21070D) which overlapped the $R \operatorname{lm} 3$ interval defined in the TC population.

During the phenotypic screening of the mapping populations with single isolates, some intermediate phenotypes (scores between 4.1 and 6.9 on the $0-9$ scale) were observed. Therefore, to test for QTL affecting the expression of the cotyledon resistance phenotype when

Table 3 Determination of A07 blackleg $R$ genes in AG-Castle and AV-Sapphire

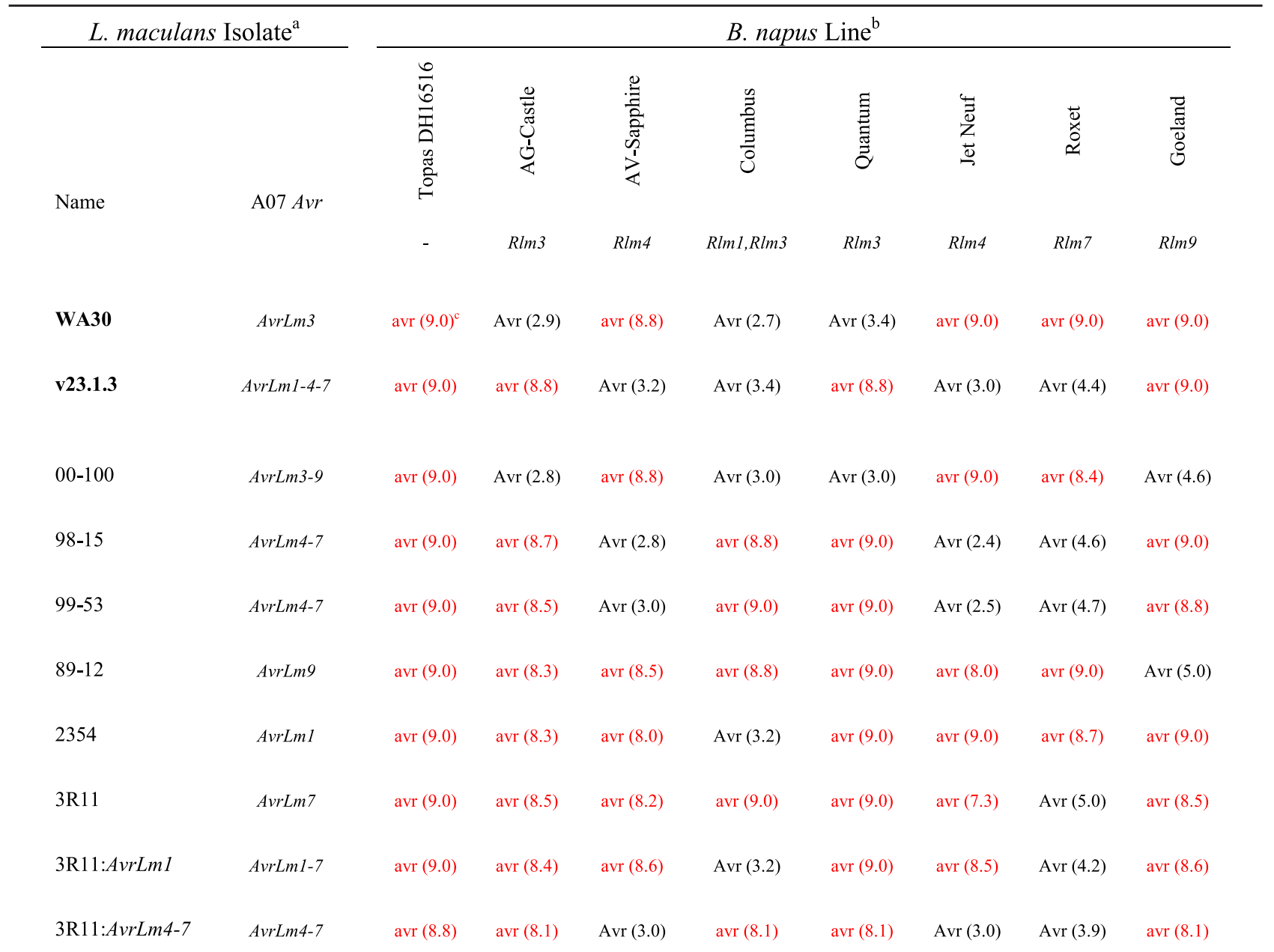

${ }^{a}$ L. maculans isolates used in this study; name followed by avirulence genes corresponding to Brassica A07 $R$ genes carried by each isolate. 3R11: AvrLm1 and 3R11: AvrLm4-7 are transgenic isolates of 3R11 carrying additions of AvrLm1 and AvrLm4-7, respectively. Isolates in bold were used for TC and TS population cotyledon assays ${ }^{\mathrm{b}} B$. napus lines used in the study; name followed by blackleg $R$ gene content of line

Interaction of isolate and B. napus line; avr = virulence, Avr = avirulence, followed by mean cotyledon rating (in brackets) on 0-9 scale. Entries in red indicate a virulent interaction 
challenged with single isolates of $L$. maculans, the phenotypic data was again analysed, this time as continuous data (0 to 9) rather than the discrete "Mendelised" data ('+' or '-') analysed previously. As expected, large portions of the variance were associated with each major resistance gene locus (Rlm3 and $R \operatorname{lm} 4$ ). We also detected a second significant QTL for the TC population which accounted for $9.26 \%$ of the cotyledon phenotypic variance and co-localised with the TC C06 QTL cluster (Table 2, Fig. 2).

\section{Delineation of QTL loci and identification of candidate genes}

The $0.5 \mathrm{cM}$ map interval containing the TC A01 MET S and MET II loci was flanked by the markers sN12790 and sN4638 (Fig. 2), which corresponds to a span of 49 B. napus genes (BnaA01g12170D-BnaA01g12660D). The peak MET LOD scores for each metric were flanked by the markers sR 8420 and sN4638 which corresponded to a 10 gene interval of the Darmor-bzh reference $B$. napus genome (BnaA01g12560D-BnaA01g12660D). The TS A01 QTL locus was flanked by the markers sR9228a and sN12176, representing a span of 296 genes (BnaA01g09950D to BnaA01g12910D), with peak LODs for all QTL contained with the $4.2 \mathrm{cM}$ marker interval sR6202b-sN12176 (BnaA01g10980D to BnaA01g 12910D), representing a span of 193 B. napus genes which also encapsulates the physical interval defined for the TC A01 QTL locus.

The TC population produced a cluster of single- and multi-environment QTL on chromosome A08, with peak values for all QTL positioned between 39 and $46 \mathrm{cM}$ (Table 2). The multi-environment analysis defined overlapping MET S and MET II QTL contained within a marker interval of $13.7 \mathrm{cM}$ flanked by the markers sN4513Fa and sN12352a. This interval corresponds to a region of 396 genes in the Darmor- $b z h$ reference genome (BnaA08g18290D-BnaA08g22250D).

The QTL cluster detected for the TS population on chromosome A09 was positioned within the marker interval sS2212 to sR9373, which corresponds to a physical interval of 1314 genes in the B. napus reference genome (BnaA09g22470D-BnaA09g35610D). While the peak LOD for many of the QTL, including both the MET S and MET II QTL, was positioned within the smaller sR6410sR9373 marker interval, the physical region could not be refined any further using the B. napus reference genome, as sR6410 was assigned to the 'chrA09_random' molecule which is not incorporated into the main A09 chromosome build. However, by using the $B$. rapa Chiifu A genome reference sequence [41], we identified homology between sR6410 and the B. rapa A09 gene Bra006927 and used a neighbouring gene (Bra006925) to determine an approximate $B$. napus physical position for this gene as equivalent to BnaA09g32910D of B. napus. This produced a physical delineation for the A09 QTL peak interval of approximately 270 genes (BnaA09g32910D-BnaA09g35610D).

QTL clusters were also detected on chromosome C06 in each population, though poor resolution of these linkage groups hampered precise delineation of the support intervals. The TS C06 QTL support interval was delimited to a 2998 gene span of the B. napus genome (BnaC06g05190D-BnaC06g35170D) between the markers sS2486 and sN5088F while in the TC population, a $B$. napus physical interval could not be defined as the upper flanking marker for the TC C06 support interval (DArT marker brPb-841355) provide a match to the unincorporated 'chrUn_random' molecule. We were, however, able to define a syntenic physical interval in the $B$. oleracea reference genome [42] of approximately $9 \mathrm{Mb}$ on C06 $(1,797,307 . .10,864,498)$, which contains 1336 predicted genes.

Delineation of the TC A01, TC A08 and TS A09 QTL loci produced physical intervals sufficiently small to warrant identification of gene candidates that had potential roles in underpinning pathogen resistance QTL. The TC A01 QTL peak LOD interval spanned only ten genes, which straddled a cluster of eight genes (BnaA01g12580D12590D, BnaA01g12610D-12630D, BnaA01g12650D12670D) with homology to the Cysteine-rich ReceptorLike Protein Kinase (CRK) genes of A thaliana chromosome 4 (At4g23190, At4g23300 and At4g04570, respectively). CRKs are one of the largest super-families of receptor kinases in Arabidopsis with 44 members [43], several of which have been implicated in plant defense responses [44-49]. The TC A08 and TS A09 peak QTL intervals span 396 and 270 genes within the reference $B$. napus genome, respectively. Within these spans are several potential resistance-related genes including receptorlike proteins, a receptor-like kinase and TIR-NB-LRR homologues.

\section{Discussion}

We describe here the detection and characterisation of highly-stable quantitative resistance loci to the B. napus fungal pathogen $L$. maculans. Through multi-environment analysis we were able to define highly-heritable resistance loci effective in some of the harshest testing conditions in the world and to identify putative resistance-related genes that are located within the physically-defined QTL regions.

In performing the QTL tests over multiple environments we were able to produce estimates of heritability $\left(h^{2}\right.$ : the degree to which genetics determines phenotype) for each MET QTL (Table 2). Traditionally heritability is calculated as a function of the variance within the entire population (Table 1). While this provides an estimate of the genetic influence on the over-all phenotypic variance, this does not provide information on the environmental 
variability of individual QTL loci within the population. By calculating $h^{2}$ values for each MET QTL we can offer an estimate of how environmental variability will affect the phenotypic variation, particularly when targeting individual QTL loci in breeding programs. We also observed that in the larger TC population (242 DH lines), the $h^{2}$ values were consistently higher when MET QTL were determined using the survival metric compared to the internal infection metric, though this did not hold true for the smaller TS population (109 lines). This may be due to the influence of 'escapes' in the scoring of the field trials. While significant differences were seen in survival between the 'susceptible' Topas and 'resistant' parental cultivars AG-Castle and AV-Sapphire, the difference was not always evident with the internal infection metric (Additional file 4: Table S2, Additional file 1: Figure S1). Under Australian field conditions, infection is driven by sexual ascospores and often results in seedling death for susceptible plants [50]. This means very few susceptible plants will remain standing at the end of the growing season when internal infection is assessed. However, the remaining survivors have been enriched for escapes i.e. plants that did not develop the same level of disease due to a delay in, or lack of, exposure to the pathogen, particularly when seedling germination is not consistent. This would result in an under-estimate of internal infection for individual lines, as only a portion of the standing plants are assessed. The same effect would not be as significant in the survival metric where the entire row is counted.

The genomic location of the TC A01 QTL interval matches the previously reported position of a blackleg QTL from the Australian cultivar AG-Spectrum [19]. A recent report detailing QTL mapping in European winter oilseed rape populations [51] placed QTL from Grizzly/Bristol and Darmor/Bristol populations within a region of A01 that spans the TC A01 QTL locus defined in our study (approx. BnaA01g08200D-BnaA01g20140D). QTL on chromosome A01 have also previously been reported for several DH populations derived from Australian varieties, including AV-Sapphire [27]. Favourable alleles from the AG-Castle A08 QTL locus (BnaA08g 18290D-BnaA08g22250D) are positioned adjacent to the QlmA8_DB QTLs previously identified in the Darmor/ Bristol population, which were positioned between the SSR markers BN53449 and sR3688 (BnaA08g12480DBnaA08g17050D) on chromosome A08 [51], and to the DY A08 QTL detected in the Darmor-bzh/Yudal population $[26,52]$. Additionally, the TS A09 QTL locus from AV-Sapphire provides a near-complete overlap with the previously-reported syntenic $A$. thaliana region At3g25805At3g58680 (equivalent to approximately BnaA09g19610D to BnaA09g37480D of the B. napus genome), which was identified as syntenic to the $L m A 9$ QTL interval from the Darmor-bzh/Yudal QTL map [26]. Finally, QTLs from
Aviso and Darmor/Bristol populations were also placed within a 369 gene interval (BnaC06g31460D-BnaC06g 35150D) on lower C06 [51], which is in agreement with the large C06 QTL regions detected in both the TC and TS populations. The correlation of blackleg QTL in many of the studied $B$. napus varieties suggests the overall pool of APR genetics utilised in canola varieties world-wide may be rather limited.

Pedigree analysis for AG-Castle suggests nearly half of the variety's genetic contribution $(46.9 \%)$ is derived from Japanese material, with European material making up the bulk of the remainder (29.8\%) [53]. A study of $B$. napus germplasm diversity has shown the variety AGSpectrum is closely related to Rainbow [54], an Australian polygenic resistant variety which is also featured in the pedigree for AG-Castle [53]. The B. napus variety Major, a progenitor of the well-characterised French APR variety Darmor (Major - > Primor - > Jet Neuf - > Darmor) was grouped into a different clade than Rainbow and AGSpectrum [54], suggesting a low over-all genomic relationship between sources of APR for the Australian and European QTL studies. However, the correlation of QTL on A01, A08, A09 and C06 between Australian and French varieties demonstrates a high degree of selection and retention of these QTL after the introduction of French APR germplasm into Australian breeding programs [22], suggesting enduring efficacy of these QTL against Australian L. maculans populations. However, a "slow erosion of polygenic resistance" has been observed in Australian breeding programs [53] and suggests that future efforts should be focused on the detection and introgression of novel APR genetics from diverse Brassica germplasm rather than the continued recycling of overused QTL. Interestingly, the B. juncea line BJ168 also makes a small contribution (6.3\%) to the pedigree of AGCastle and many other Australian varieties, though none of the major $R$ genes from $B$. juncea have been introgressed into Australian cultivars [53]. These $R$ genes, present in the B genome of $B$. juncea, are potentially valuable sources of resistance for $B$. napus breeding if stably introgressed [55-57]. However, little is known of the quantitative resistance potential of the B. juncea A genome, which harbours distinct genomic diversity [58], or if $B$. juncea A genome introgressions have contributed to the pool of blackleg APR utilised in Australian germplasm, and this remains a potentially under-utilised resource for resistance genetics.

Investigation of the genomic region defined by the TC A01 peak QTL interval revealed a cluster of cysteine-rich receptor-like kinase genes (CRKs). CRKs are characterised by one or more extracellular C-X8-C-X2-C motifs (DUF26/GNK2) that likely mediate protein-protein interactions [43]. CRK genes have been shown to be induced in plants during pathogen infection [44-49], including the 
upregulation of several CRKs during infection of $B$. napus cotyledons by $L$. maculans [32]. Active early in the plant defence response to pathogens, CRKs are induced independent of other host response genes such as EDS1 or NPR1 [44, 45]. While some members of the CRK superfamily have been demonstrated to enhance resistance against pathogens by promoting hypersensitive response (HR) and salicylic acid (SA) accumulation [44, 45], the use of knock-out mutants and transient gene silencing has suggested that some other CRKs can negatively affect defense responses, acting in a quantitative fashion $[47,48]$. CRKs have also been shown to have a role in symbiotic plant-microbe interactions, with the symCRK gene of the legume Medicago truncatula being required for maintenance of functional nodules and the suppression of host defense during the symbiotic process [59]. Recently it was demonstrated that over-expression of some Arabidopsis CRKs enhanced PTI defense responses, such as oxidative burst, stomatal closure and callose deposition, towards the bacterial pathogen Pseudomonas syringae pv. tomato DC3000. Additionally, these proteins were shown to associate with the pattern-recognition receptor FLAGELLIN SENSING2 (FLS2) at the plasma membrane [60], an essential component of the bacterial pathogen PAMP response [61]. A second study, profiling a near-complete set of Arabidopsis CRK mutants, showed some CRKs respond to fungal chitin, a common PTI elicitor [62, 63], and play a role in regulating stomatal closure [64]. The roles of CRKs in quantitative plant defense responses suggests the cluster of CRK genes colocalised with the TC A01 QTL interval offer viable candidate genes for blackleg APR. Further study of these genes, including their temporal and spatial regulation in response to L. maculans infection, is currently underway. While the other QTL intervals defined in this study were not small enough to warrant extensive candidate gene studies, other CRK homologues were found with the TS A09 (BnaA09g 23770D) and TS C06 (BnaC06g31680) intervals.

While no stable QTL were detected in which the resistant allele originated from the Topas "susceptible" parent, it should be noted that in most environments Topas had a higher survival percentage than the commonly-used susceptible line Westar (Additional file 4: Table S2). Under the disease nursery conditions in Australia, the Westar rows showed near-complete seedling death, often leaving no plants at all at the end of the growing season, while the Topas rows still produced some adult plants, though most were dead or highly cankered at the time of rating. It is conceivable that Topas contains some small degree of APR common to both AG-Castle and AV-Sapphire, and perhaps many other B. napus cultivars, which would not be detectable as QTL in these populations. While some Topas-derived QTL were detected during the study, these were always specific to a single testing environment, usually accounted for less than $5 \%$ of the variance and were not associated with any significant MET QTL (Additional file 2: Table S1). Under the extremely high disease pressure of the Horsham 2012 trial, both Topas and Westar had $0 \%$ survival (Additional file 4: Table S2, Additional file 1: Figure S1), so any minor APR that may have been expressed by Topas was rendered completely ineffective under those conditions.

Our single-isolate characterisation of the TC and TS populations also delimited the physical positions of the major $R$ genes $R \operatorname{lm} 3$ and $R \operatorname{lm} 4$ to intervals of $3.5 \mathrm{Mb}$ and 3.9 Mb, respectively, on chromosome A07. While these genes have been known to cluster genetically, along with $\operatorname{Rlm} 7$ and $R \operatorname{lm} 9$, on A07 for over a decade [38], this is the first report to define a physical location for this cluster in the B. napus genome. Previously, Raman et al. (2012c) positioned the Rlm4 gene (from $B$. napus variety Skipton), assessed at both the cotyledon and adult plant stages, to a $24.8 \mathrm{cM}$ interval of A07 using SNP markers. While the physical interval relative to $B$. napus was not defined in this study, several SNPs corresponding to approximately 15.9-16.9 Mb ( BnaA07g20250D-BnaA07g21900D) on B. napus A07 were associated with the Rlm4 locus [65]. This location would be in agreement with the $R \operatorname{lm} 4$ physical interval defined for the TS population in this study (BnaA07g14030D-BnaA07g21070D) and the cosegregation of the locus with the SSR marker sR12173 (BnaA07g20910D).

Neither Rlm3 nor Rlm4 were found to be associated with QTL detected in the field studies, suggesting a high degree of virulence towards both $R$ genes in the populations of $L$. maculans infesting the disease nurseries. This result was not unexpected, as high rates of virulence towards $R \operatorname{lm} 3$ [66] and $R \operatorname{lm} 4$ [23] have been previously reported for Australian L. maculans isolates. Analysing the cotyledon reactions to single $L$. maculans isolates avirulent towards the respective $R$ genes produced high LOD, high variance 'CotQTL' loci associated with each gene, as expected. An additional cotyledon-responsive interaction with the isolate WA30 was also demonstrated in the TC population which accounted for $9.26 \%$ of the cotyledon phenotypic variation and localised to the C06 QTL interval (Fig. 2, Table 2). No additional significant cotyledon-responsive QTL were detected in the TS population, which also contains a C06 QTL locus, using isolate v23.1.3. Previous studies have observed a significant difference in $L$. maculans cotyledon lesion development between APR and non-APR varieties [18]. Cotyledon-expressed QTL similar to the one detected in our study have also previously been defined in the SASDH (Skipton/ AG-Spectrum) population and localised on chromosomes A01 and A10, though only the A01 QTL was associated with field resistance in the 
adult plant. These cotyledon QTL interactions also appeared to be race-specific, having been detected with the L. maculans isolate 06MGPP041 but not isolate 04MGPS021 [19]. Blackleg resistance at seedling and adult stages of the host have often been viewed as separate mechanisms; the former governed by racespecific $R$ gene interaction in cotyledons and leaves, and the latter by non-specific QTL active in the stem. The detection of common QTL loci in both seedling (cotyledon) and adult plant (field) tests, and the differential interaction of individual $L$. maculans isolates to those QTL, suggests that such distinctions are not always valid. The associated resistance response is likely triggered early in the interaction between host and pathogen, activated long before entry of the pathogen into the stem tissues of the plant. The action of these QTL may be to impair the initial infection process, rather than to actively resist invasion of adult stem tissues, leading to delayed development of stem canker symptoms in the adult plant. If the quantitative blackleg resistance found in B. napus is truly "nonrace specific" then the entirety of the environmental variance observed in field trials can be attributed to differences in the physical environmental factors (rainfall, temperature etc.) affecting each trial. However, if $L$. maculans isolates react differentially to QTL, then a large component of the environmental variance could be explained by variation in the pathogen populations used in the trials, both between sites and years. Alternatively, as it has been previously shown that $B$. napus blackleg $R$ genes require other partner proteins in order to successful trigger the resistance response to L. maculans [29, 67], minor QTL detected during cotyledon tests may represent either allelic variation or altered transcriptional patterns for $R$ gene interacting proteins. Further dissection of the temporal and spatial effects of quantitative resistance responses during $L$. maculans infection, as well as the effectiveness of these responses against a number of diverse isolates, will aid in bettering our overall understanding of the blackleg resistance response and how best to utilise the available genetics in crop protection.

\section{Conclusions}

As pathogen populations become enriched for virulence against commonly-deployed $R$ genes, alternative resistance genetics need to be incorporated into crop breeding programs in order to maintain global agricultural production. The identification and mapping of QTL is required for the efficient introgression of stable resistance genetics in modern MAS breeding programs, enabling the effective utilisation of quantitative genetics in plant protection. In this study we have delineated four stable blackleg resistance QTL through multi-environment QTL analysis. We have identified a cluster of cysteine-rich receptor-like kinase genes associated with a significant field-derived QTL, suggesting a role for these genes in quantitative defense responses to L. maculans. The genomic delineation of effective blackleg resistance QTL described in this work should benefit resistance breeding efforts, and further the investigation into the identity of genes controlling APR in Brassica germplasm.

\section{Methods \\ Plant populations}

$\mathrm{F}_{1}$ seedlings, produced by crossing the Swedish springtype, blackleg-susceptible $B$. napus variety Topas with the Australian blackleg-resistant varieties AG-Castle and AV-Sapphire, were used to produce DH populations via microspore culture by the method of Ferrie et al. [68] using modified Lichter medium (NLN) with $13 \%$ sucrose. The Topas/AG-Castle DH population (TC) utilised for this study consisted of a total of $242 \mathrm{DH}$ lines from two sub-populations of 139 and $103 \mathrm{DH}$ lines, with each sub-population produced from a single $F_{1}$ plant. The Topas/AV-Sapphire DH population (TS) contained a total of 109 lines, also consisting of two sub-populations of 66 and 43 DH lines. Seed of parental and DH lines used in the QTL trials was produced in tented field plots in Saskatoon, Canada during the 2007 (TS) and 2008 (TC) summer growing seasons, packaged into replicates of 100 seeds per line and sent to Australia for testing field resistance to blackleg. The Topas B. napus line was sourced from the AAFC Saskatoon collection. AG-Castle and AV-Sapphire lines were provided by DEDJTR Victoria (formerly Department of Primary Industries).

\section{Field characterisation of $\mathrm{DH}$ populations}

The parental lines and their respective DH populations, along with several $B$. napus variety checks (Additional file 4: Table S2) were grown May through November in blackleg disease nurseries situated at Wagga Wagga (New South Wales) and two sites (Wonwondah and Green Lake) south of Horsham (Victoria) between 2008 and 2012 (Fig. 1). Rainfall differed by an average of $103.2 \mathrm{~mm} /$ year over the three trial years at each site, with the Wagga Wagga site receiving an average $38 \%$ more precipitation (Additional file 5: Table S3). Each trial was planted in a randomised block design with 3 to 4 replicates per line. The TC population was tested in three separate environments; both Wagga Wagga and Horsham (Wonwondah site) in 2009 (3 replicates per trial) and once more at Wagga Wagga in 2010 (4 replicates). The TS population was tested in four environments; Horsham (Wonwondah site), 2008 and Wagga Wagga, 2009 as 3-replicate trials and Wagga Wagga, 2011 and Horsham (Green Lake site), 2012 as 4replicate trials. Emergence counts were made 4-5 weeks after sowing. For trials at the Horsham sites, local diseased canola stubble, collected in the previous year, was 
spread as the source of $L$. maculans inoculum, while at Wagga Wagga the trials were sown into in situ canola stubble from the preceding season as previously described [19]. At the end of the growing season trials were assessed for blackleg disease using both survival percent (S) and internal infection (II) metrics. S was calculated for each row as (Standing plants at end of season/Emergence count)*100, II determined by examining 5-20 random plants per row for internal infection after cutting the stem at the crown and rating fungal discoloration on a $0-5$ scale $(0=$ no infection, $1=1-25 \%$ infection, $2=26-50 \%$ infection, $3=51-75 \%$ infection and $4=76-99 \%$ infection and $5=100 \%$ infection $/ \mathrm{missing}$ plant) and median scores were calculated for each entry and metric using Microsoft Excel. Statistical analysis of populations and plotting of distribution histograms were performed using R software [69].

Selected lines representative of the 3 major QTL identified from the TC DH population in this study, both singularly and in combination, were also entered into Saskatchewan disease nurseries at Melfort (2012) and Saskatoon (2013) in attempt to test the efficacy of the QTL resistance against Canadian populations of $L$. maculans. However, neither site produced sufficient disease pressure to assess the resistance, with Westar control entries presenting average internal infections of less than $25 \%$ at the end of the growing season.

\section{Single-isolate characterisation}

Each DH line in the TC and TS populations were characterised for the presence or absence of hypersensitive response during infection by the single spore-derived $L$. maculans isolates WA30 (Rimmer Collection, AAFC Saskatoon) or v23.1.3 (INRA-Bioger collection, France), respectively, via cotyledon infection tests. Four plants for each line were scored at 14 days post-infection on a $0-9$ scale as described by Larkan et al. [13]. Further differential pathology testing, using isolates of $L$. maculans varying in their avirulence gene profiles (00-100, 98-15, 99-53, 8912, 2354-Rimmer Collection, AAFC Saskatoon; 3R11Howlett Lab, University of Melbourne, Australia, 3R11: AvrLm1 [13]) was performed with the parental lines and the $R$ gene control lines Topas DH16516 (a DH line of Topas with no effective blackleg resistance), Columbus (Rlm1, Rlm3), Quantum (Rlm3), Jet Neuf (Rlm4), Roxet $(R \operatorname{lm} 7)$ and Goeland $(R \operatorname{lm} 9)$ in order to produce a hypothesis for the likely $R$ gene content of each population (Table 3). Inoculum for the tests was produced as described previously [70]. Spores were diluted to $2 \times 10^{7}$ spores $/ \mathrm{mL}$ and $10 \mu \mathrm{L}$ was used to inoculate each of four small wounds on each plant (one wound at the centre of each cotyledon lobe).

The transgenic isolate 3R11: AvrLm4-7 was produced by Agrobacterium transformation [71]. Briefly, a 2205 bp amplicon was produced from the isolate v23.1.3 (primers: GW-AvrLm47G-Fc; GGGGACAAGTTTGTA CAAAAAAGCAGGCTTCCTCTGGTAAGGAAGGTT TACCAATTATACACCT, GW-AvrLm47G-Rb; GGGG ACCACTTTGTACAAGAAAGCTGGGTCGGCGGTA GATTTGCTACTAAAAGGTAACTTT) which spanned 1213 bp upstream and 514 bp downstream of the $L$ maculans AvrLm4-7 CDS [72] and inserted into the Gatewaycompatible fungal transformation vector pNL11 prior to transformation of the L. maculans isolate $3 R 11$ as previously described [13].

\section{Map construction and QTL identification}

The TC and TS populations were characterised using 225 and 212 simple sequence repeat (SSR) markers, respectively, as described previously [73]. A subset of the TC population had previously been characterised using 279 DArT markers as part of a consensus map of $B$. napus [74], and this data was also incorporated into the TC map to provide additional resolution and to allow orientation to other maps (503 markers total, grouped into 360 bins). Linkage map construction and inclusive composite interval mapping (ICIM) [75] was performed using QTL IciMapping version 3.3 (http://www.isbreeding. net). Prior to final map construction severely distorted markers (Chi-square Test, $p<0.001$ ) were excluded. A threshold of LOD 6 was applied for initial grouping of markers. LGs consisting of $>3$ markers were anchored to chromosomes based on BLAST-like alignment tool (BLAT) analysis of SSR marker sequences to the published B. napus genome [37] before recalculating LGs at LOD 3. Marker ordering was performed using nearest neighbour algorithm and rippling was performed once using the sum of adjacent recombination fractions (SARF) criterion. For QTL identification, the 'ICIM-ADD' and 'ICIM-EPI' functions of the software were utilised to investigate both additive and digenic epistatic QTL $[76,77]$ expressed in each environment and for each scoring metric (S and II). Permutation tests (1000 permutations, $95 \%$ confidence level, $1 \mathrm{cM}$ interval) were performed for each scoring metric in each environment to determine significant LOD thresholds. The precision of QTL positions were then improved by reanalysing the data using the determined LOD thresholds and a smaller $(0.5 \mathrm{cM})$ scan interval. The 'MultiEnvironment Trials' (MET) function of the software was also utilised to determine the consensus positions for the major QTL and to assess the genotype by environment (GxE) interactions. QTL identified from individual environments were considered significant if they exceeded the LOD significance threshold and accounted for $>5 \%$ of the variance. MET QTL were considered significant if they accounted for $>5 \%$ of the variance and had a heritability $\left(h^{2}=\sigma^{2}\right.$ (Additive) $/ \sigma^{2}($ Total $\left.)\right)>0.5$. QTLs positions were defined by both support and peak marker intervals, with 
support intervals representing the map interval in which the LOD exceeded the calculated LOD threshold (for single-environment QTL), or peak LOD-1 (for multienvironment analyses). The data sets for each population were analysed both as total populations and as their separate component sub-populations. Final map figures were produced using Mapchart 2.2 [78] and Microsoft PowerPoint software. To determine the location of the major QTL relative to the B. napus reference Darmor-bzh genome [37] the target sequences for the flanking SSR markers were obtained from the AAFC Brassica MAST Database (http://aafc-aac.usask.ca/BrassicaMAST/) and used to search the public B. napus genome database (http://www.genoscope.cns.fr) using default BLAT search settings.

\section{Additional files}

Additional file 1: Figure S1. Distribution of Median Survival and Internal Infection Percentages. Distribution of scores for survival (green bars) and internal infection (red bars) given for each population (TC or TS) in each trial. Environment names given as location $(H=$ Horsham, W = Wagga Wagga), year $(08-12=2008-2012)$. Green and red lines indicate mean survival and internal infection percentages for parental lines, respectively. (PNG $127 \mathrm{~kb}$ )

Additional file 2: Table S1. All single- and multi-environment QTL (LOD > 2.5). (DOCX $43 \mathrm{~kb})$

Additional file 3: Figure S2. Transgenic complementation of R/m4 in AV-Sapphire. Phenotypic interaction of isolates 00-100 (avrLm4-7), v23.1.3 (AvrLm4-7), 3R11 (AvrLm7) and transgenic isolate 3R11: AvrLm4-7 (AvrLm4-7) with $B$. napus lines Topas (no blackleg $R$ genes) and AV-Sapphire (R/m4). (PNG 1656 kb)

Additional file 4: Table S2. Survival and internal infection of control $B$. napus lines in each environment. (DOCX $16 \mathrm{~kb}$ )

Additional file 5: Table S3. Rainfall ( $\mathrm{mm}$ ) for trial sites over growing season (May-Nov). (DOCX $15 \mathrm{~kb}$ )

\section{Acknowledgments}

The authors would like to thank Sarah Kuzmicz, Kyla Horner, Sigrun Gulden, Christine Hammond, Helen Lui, Catherine Guenther and Stephanie McMillan (AAFC, Saskatoon, Canada), Ray Cowley and David Roberts, Peter Heffernan, Peter Deane and Brian (NSWDPI, Wagga Wagga, Australia), Shanoor Hossain and Sandra Maybery (DEDJTR, Horsham, Australia) for assistance with technical aspects of the project. We would also like to thank Regine Delourme (INRA, Rennes, France) for personal communications regarding blackleg QTL, Thierry Rouxel (INRA-Bioger, France) for providing the reference L. maculans isolate V23.1.3 and Angela Van de Wouw \& Barbara Howlett (University of Melbourne, Australia) for providing the isolate 3R11.

\section{Funding}

This work was funded by the AAFC-Industry Blackleg Consortium II (members include Advanta Seeds Pty Ltd, Agriculture Victoria Services, Bayer, Crop Production Services Canada, Dow AgroSciences, KWS SAAT AG and NSW Department of Primary Industries).

\section{Availability of data and materials}

All data supporting the findings is contained within the manuscript and supplemental data sections. Details of the SSR markers used in the study are publically available online as described in the methods section. DH populations used in the study and data on individual $\mathrm{DH}$ lines remain the property of Agriculture \& Agri-Food Canada.

\section{Authors' contributions}

D Lydiate and S Rimmer conceived of the study. D Lydiate, S Rimmer, FY and $M B$ participated in the project coordination. NL, HR, DB, D Luckett, WB, NW and PS organised and/or performed field trials. NL performed the genetic mapping and genomic analysis. HR and RR provided DArT marker analysis. NL and S Robinson performed statistical and QTL analysis. NL, MB and HR drafted the manuscript. All authors except S Rimmer (deceased) read and approved the final manuscript.

\section{Competing interests}

The authors declare no competing interests in regards to the work presented here.

\section{Consent for publication}

Not applicable.

\section{Ethics approval and consent to participate}

Not applicable.

\section{Author details}

${ }^{1}$ Saskatoon Research Centre, Agriculture and Agri-Food Canada, Saskatoon, SK, S7N 0X2, Canada. ${ }^{2}$ Armatus Genetics Inc, Saskatoon, SK, S7W 0C9, Canada. ${ }^{3}$ Graham Centre for Agricultural Innovation (an alliance between Charles Sturt University and NSW Department of Primary Industries), Wagga Wagga Agricultural Institute, Wagga Wagga NSW 2650, Australia. ${ }^{4}$ Department of Economic Development, Jobs, Transport and Resources, Grains Innovation Park, Horsham, VIC, 3400, Australia. ${ }^{5}$ Seednet Australia, Golf Course Road, Horsham, VIC, 3402, Australia. ' ${ }^{6}$ epartment of Economic Development, Jobs, Transport and Resources, Centre for AgriBioscience, La Trobe University, Bundoora, VIC, 3083, Australia. ${ }^{7}$ Faculty of Veterinary and Agricultural Sciences, University of Melbourne, Melbourne, VIC, 3010, Australia.

Received: 18 May 2016 Accepted: 17 August 2016

Published online: 24 August 2016

\section{References}

1. Bent AF, Mackey D. Elicitors, effectors, and R genes: The new paradigm and a lifetime supply of questions. Annu Rev Phytopathol. 2008:45:399-436.

2. Wu J, Cai G, Tu J, Li L, Liu S, Luo X, Zhou L, Fan C, Zhou Y. Identification of QTLS for resistance to Sclerotinia Stem Rot and BnaC. IGMT5.a as a candidate gene of the major resistant QTL SRC6 in Brassica napus. PLoS ONE. 2013;8(7).e67740.

3. Yin X, Yi B, Chen W, Zhang W, Tu J, Fernando WGD, Fu T. Mapping of QTLs detected in a Brassica napus DH population for resistance to Sclerotinia sclerotiorum in multiple environments. Euphytica. 2010;173(1):25-35.

4. Zhao J, Udall JA, Quijada PA, Grau CR, Meng J, Osborn TC. Quantitative trait loci for resistance to Sclerotinia sclerotiorum and its association with a homeologous non-reciprocal transposition in Brassica napus L. Theor Appl Genet. 2006;112(3):509-16.

5. Obermeier C, Hossain MA, Snowdon R, Knüfer J, von Tiedemann A, Friedt W. Genetic analysis of phenylpropanoid metabolites associated with resistance against Verticillium longisporum in Brassica napus. Mol Breed. 2013;31(2):347-61.

6. Rygulla W, Snowdon RJ, Friedt W, Happstadius I, Cheung WY, Chen D. Identification of quantitative trait loci for resistance against Verticillium longisporum in oilseed rape (Brassica napus). Phytopathology. 2008;98(2):215-21.

7. Eynck C, Koopmann B, Karlovsky P, Von Tiedemann A. Internal resistance in winter oilseed rape inhibits systemic spread of the vascular pathogen Verticillium longisporum. Phytopathology. 2009;99(7):802-11.

8. Howlett BJ, Idnurm A, Pedras MSC. Leptosphaeria maculans, the causal agent of blackleg disease of Brassicas. Fungal Genet Biol. 2001;33(1):1-14.

9. Raman H, Raman R, Larkan N. Genetic dissection of blackleg resistance Loci in rapeseed (Brassica napus L.). Andersen SB, editor. Plant breeding from laboratories to fields. InTech; 2013. doi: 10.5772/53611. Available from: http://www.intechopen.com/books/plant-breeding-from-laboratories-tofields/genetic-dissection-of-blackleg-resistance-loci-in-rapeseed-brassicanapus-I-.

10. Rouxel T, Penaud A, Pinochet X, Brun H, Gout L, Delourme R, Schmit J, Balesdent MH. A 10-year survey of populations of Leptosphaeria maculans in France indicates a rapid adaptation towards the $R / \mathrm{m} 1$ resistance gene of oilseed rape. Eur J Plant Pathol. 2003;109(8):871-81. 
11. Daverdin G, Rouxel T, Gout L, Aubertot JN, Fudal I, Meyer M, Parlange F, Carpezat J, Balesdent $\mathrm{MH}$. Genome structure and reproductive behaviour influence the evolutionary potential of a fungal phytopathogen. PLoS Pathog. 2012;8(11).e1003020.

12. Sprague SJ, Marcroft SJ, Hayden HL, Howlett BJ. Major gene resistance to blackleg in Brassica napus overcome within three years of commercial production in Southeastern Australia. Plant Dis. 2006;90(2):190-8.

13. Larkan NJ, Lydiate DJ, Parkin IAP, Nelson MN, Epp DJ, Cowling WA, Rimmer $\mathrm{SR}$, Borhan $\mathrm{MH}$. The Brassica napus blackleg resistance gene LepR3 encodes a receptor-like protein triggered by the Leptosphaeria maculans effector AVRLM1. New Phytol. 2013;197(2):595-605.

14. Delourme R, Chevre AM, Brun H, Rouxel T, Balesdent MH, Dias JS, Salisbury $P$, Renard M, Rimmer SR. Major gene and polygenic resistance to Leptosphaeria maculans in oilseed rape (Brassica napus). Eur J Plant Pathol. 2006;114(1):41-52.

15. Brun $H$, Chèvre AM, Fitt BD, Powers S, Besnard AL, Ermel M, Huteau V, Marquer B, Eber F, Renard M, et al. Quantitative resistance increases the durability of qualitative resistance to Leptosphaeria maculans in Brassica napus. New Phytol. 2010;185(1):285-99.

16. Delourme R, Bousset L, Ermel E, Duffé P, Besnard AL, Marquer B, Fudal I, Linglin J, Chadœuf J, Brun H. Quantitative resistance affects the speed of frequency increase but not the diversity of the virulence alleles overcoming a major resistance gene to Leptosphaeria maculans in oilseed rape. Infect Genet Evol. 2014:27:490-99.

17. Rimmer SR. Resistance genes to Leptosphaeria maculans in Brassica napus. Can J Plant Pathol. 2006:28:S288-97.

18. Huang YJ, Qi A, King GJ, Fitt BDL. Assessing Quantitative resistance against Leptosphaeria maculans (phoma stem canker) in Brassica napus (oilseed rape) in young plants. PLOS ONE. 2014;9(1).e84924.

19. Raman R, Taylor B, Marcroft S, Stiller J, Eckermann P, Coombes N, Rehman A, Lindbeck K, Luckett D, Wratten N, et al. Molecular mapping of qualitative and quantitative loci for resistance to Leptosphaeria maculans causing blackleg disease in canola (Brassica napus L.). Theor Appl Genet. 2012;125(2):405-18.

20. Pang ECK, Halloran GM. The genetics of blackleg [Leptosphaeria maculans (Desm.) Ces. et De Not.] resistance in rapeseed (Brassica napus L). II. Seedling and adult-plant resistance as quantitative traits. Theor Appl Genet. 1996;93(5-6):941-9.

21. Rouxel T, Willner E, Coudard L, Balesdent MH. Screening and identification of resistance to Leptosphaeria maculans (stem canker) in Brassica napus accessions. Euphytica. 2003;133(2):219-31.

22. Salisbury P, Wratten N. Brassica napus Breeding. In: Salisbury PA, Potter TD, McDonald G, Green AG, editors. Canola in Australia-The First Thirty Years. Gosford: The Regional Institute Ltd; 1999. p. 29-36.

23. Marcroft SJ, Elliott VL, Cozijnsen AJ, Salisbury PA, Howlett BJ, Van De Wouw AP. Identifying resistance genes to Leptosphaeria maculans in Australian Brassica napus cultivars based on reactions to isolates with known avirulence genotypes. Crop Pasture Sci. 2012;63(4):338-50.

24. Pilet ML, Delourme R, Foisset N, Renard M. Identification of loci contributing to quantitative field resistance to blackleg disease, causal agent Leptosphaeria maculans (Desm.) Ces. et de Not., in Winter rapeseed (Brassica napus L.). Theor Appl Genet. 1998:96(1):23-30.

25. Pilet ML, Duplan G, Archipiano M, Barret P, Baron C, Horvais R, Tanguy X, Lucas MO, Renard M, Delourme R. Stability of QTL for field resistance to blackleg across two genetic backgrounds in oilseed rape. Crop Sci. 2001:41(1):197-205.

26. Delourme R, Piel N, Horvais R, Pouilly N, Domin C, Vallée P, Falentin C, Manzanares-Dauleux MJ, Renard M. Molecular and phenotypic characterization of near isogenic lines at QTL for quantitative resistance to Leptosphaeria maculans in oilseed rape (Brassica napus L.). Theor Appl Genet. 2008;117(7):1055-67.

27. Kaur S, Cogan NOI, Ye G, Baillie RC, Hand ML, Ling AE, McGearey AK, Kaur J, Hopkins CJ, Todorovic M, et al. Genetic map construction and QTL mapping of resistance to blackleg (Leptosphaeria maculans) disease in Australian canola (Brassica napus L.) cultivars. Theor Appl Genet. 2009;120(1):71-83.

28. Ghanbarnia K, Fudal I, Larkan NJ, Links MG, Balesdent M-H, Profotova B, Fernando WGD, Rouxel T, Borhan MH. Rapid identification of the Leptosphaeria maculans avirulence gene AvrLm2 using an intraspecific comparative genomics approach. Mol Plant Pathol. 2015;16(7):699-709.

29. Larkan NJ, Ma L, Borhan MH. The Brassica napus receptor-like protein RLM2 is encoded by a second allele of the LepR3/R/m2 blackleg resistance locus. Plant Biotechnol J. 2015;13(7):983-92.
30. Li H, Kuo J, Barbetti MJ, Sivasithamparam K. Difference in the responses of stem tissues of spring-type Brassica napus cultivars with polygenic resistance and single dominant gene-based resistance to inoculation with Leptosphaeria maculans. Can J Bot. 2007:85:191-203.

31. Šašek V, Nováková $M$, Jindřichová B, Bóka K, Valentová O, Burketová L. Recognition of avirulence gene AvrLm 1 from hemibiotrophic ascomycete Leptosphaeria maculans triggers salicylic acid and ethylene signaling in Brassica napus. Mol Plant Microbe Interact. 2012;25(9):1238-50.

32. Haddadi $\mathrm{P}, \mathrm{Ma}$ L, Wang H, Borhan MH. Genome-wide transcriptome analyses provides insights into the lifestyle transition and effector repertoire of Leptosphaeria maculans during colonization of B. napus seedlings. Mol Plant Pathol. 2015. (in press). doi:10.1111/mpp.12356

33. Corina Vlot A, Dempsey DA, Klessig DF. Salicylic acid, a multifaceted hormone to combat disease. Annu Rev Phytopathol. 2009;47:177-206.

34. Kim PD, Šašek V, Burketová L, Čopíková J, Synytsya A, Jindřichová B, et al. Cell wall components of Leptosphaeria maculans enhance resistance of Brassica napus. J Agric Food Chem. 2013;61(22):5207-14.

35. Lowe RGT, Cassin A, Grandaubert J, Clark BL, Van De Wouw AP, Rouxel T, Howlett BJ. Genomes and transcriptomes of partners in plant-fungalinteractions between canola (Brassica napus) and two Leptosphaeria species. PLoS ONE. 2014;9(7):e103098.

36. Huang YJ, Pirie EJ, Evans N, Delourme R, King GJ, Fitt BDL. Quantitative resistance to symptomless growth of Leptosphaeria maculans (phoma stem canker) in Brassica napus (oilseed rape). Plant Pathol. 2009;58(2):314-23.

37. Chalhoub B, Denoeud F, Liu S, Parkin IAP, Tang H, Wang X, Chiquet J, Belcram H, Tong C, Samans B, et al. Early allopolyploid evolution in the post-Neolithic Brassica napus oilseed genome. Science. 2014;345(6199):950-3.

38. Delourme R, Pilet-Nayel ML, Archipiano M, Horvais R, Tanguy X, Rouxel T, Brun H, Renard M, Balesdent MH. A cluster of major specific resistance genes to Leptosphaeria maculans in Brassica napus. Phytopathology. 2004; 94(6):578-83.

39. Raman R, Taylor B, Lindbeck K, Coombes N, Barbulescu D, Salisbury P, Raman H. Molecular mapping and validation of R/ml gene for resistance to Leptosphaeria maculans in canola (Brassica napus L). Crop Pasture Sci. 2012;63(10):1007-17.

40. Leflon M, Brun H, Eber F, Delourme R, Lucas MO, Vallée P, Ermel M, Balesdent $\mathrm{MH}$, Chèvre AM. Detection, introgression and localization of genes conferring specific resistance to Leptosphaeria maculans from Brassica rapa into B. napus. Theor Appl Genet. 2007;115(7):897-906.

41. Wang X, Wang H, Wang J, Sun R, Wu J, Liu S, Bai Y, Mun J, Bancroft I, Cheng $F$, et al. The genome of the mesopolyploid crop species Brassica rapa. Nat Genet. 2011;43(10):1035-9.

42. Liu S, Liu Y, Yang X, Tong C, Edwards D, Parkin IAP, Zhao M, Ma J, Yu J, Huang $S$, et al. The Brassica oleracea genome reveals the asymmetrical evolution of polyploid genomes. Nat Commun. 2014;5:3930.

43. Chen Z. A superfamily of proteins with novel cysteine-rich repeats. Plant Physiol. 2001;126(2):473-6.

44. Acharya BR, Raina S, Maqbool SB, Jagadeeswaran G, Mosher SL, Appel HM, Schultz JC, Klessig DF, Raina R. Overexpression of CRK13, an Arabidopsis cysteine-rich receptor-like kinase, results in enhanced resistance to Pseudomonas syringae. Plant J. 2007;50(3):488-99.

45. Chen K, Du L, Chen Z. Sensitization of defense responses and activation of programmed cell death by a pathogen-induced receptor-like protein kinase in Arabidopsis. Plant Mol Biol. 2003;53(1-2):61-74.

46. Czernic P, Visser B, Sun W, Savouré A, Deslandes L, Marco Y, Van Montagu $M$, Verbruggen N. Characterization of an Arabidopsis thaliana receptor-like protein kinase gene activated by oxidative stress and pathogen attack. Plant J. 1999;18(3):321-7.

47. Ederli L, Madeo L, Calderini O, Gehring C, Moretti C, Buonaurio R, Paolocci F, Pasqualini S. The Arabidopsis thaliana cysteine-rich receptor-like kinase CRK20 modulates host responses to Pseudomonas syringae pv. tomato DC3000 infection. J Plant Physiol. 2011;168(15):1784-94.

48. Rayapuram C, Jensen MK, Maiser F, Shanir JV, Hornshøj H, Rung JH, Gregersen PL, Schweizer P, Collinge DB, Lyngkjær MF. Regulation of basal resistance by a powdery mildew-induced cysteine-rich receptor-like protein kinase in barley. Mol Plant Pathol. 2012;13(2):135-47.

49. Wrzaczek M, Brosché M, Salojärvi J, Kangasjärvi S, Idänheimo N, Mersmann S, Robatzek S, Karpiński S, Karpińska B, Kangasjärvi J. Transcriptional regulation of the CRK/DUF26 group of Receptor-like protein kinases by ozone and plant hormones in Arabidopsis. BMC Plant Biol. 2010;10:95.

50. Li H, Sivasithamparam K, Barbetti MJ. Soilborne ascospores and pycnidiospores of Leptosphaeria maculans can contribute significantly to 
blackleg disease epidemiology in oilseed rape (Brassica napus) in Western Australia. Australasian Plant Pathology. 2007;36(5):439-44.

51. Jestin C, Bardol N, Lodé M, Duffé P, Domin C, Vallée P, Mangin B, Manzanares-Dauleux MJ, Delourme R. Connected populations for detecting quantitative resistance factors to phoma stem canker in oilseed rape (Brassica napus L.). Mol Breed. 2015:35(8):1-16.

52. Huang YJ, Jestin C, Welham SJ, King GJ, Manzanares-Dauleux MJ, Fitt BDL, Delourme R: Identification of environmentally stable QTL for resistance against Leptosphaeria maculans in oilseed rape (Brassica napus). Theor Appl Genet. 2015:1-12.

53. Cowling WA. Genetic diversity in Australian canola and implications for crop breeding for changing future environments. Field Crop Res. 2007;104(1-3):103-11.

54. Raman H, Raman R, Nelson MN, Aslam MN, Rajasekaran R, Wratten N, Cowling WA, Kilian A, Sharpe AG, Schondelmaier J. Diversity array technology markers: Genetic diversity analyses and linkage map construction in rapeseed (Brassica napus L.). DNA Res. 2012;19(1):51-65.

55. Brun H, Levivier S, Somda I, Ruer D, Renard M, Chevre AM. A field method for evaluating the potential durability of new resistance sources: Application to the Leptosphaeria maculans-Brassica napus pathosystem. Phytopathology. 2000;90(9):961-6.

56. Chen S, Nelson MN, Chèvre AM, Jenczewski E, Li Z, Mason AS, Meng J, Plummer JA, Pradhan A, Siddique KHM, et al. Trigenomic bridges for Brassica improvement. Crit Rev Plant Sci. 2011;30(6):525-47.

57. Chèvre AM, Brun $H$, Eber $F$, Letanneur JC, Vallee $P$, Ermel $M$, Glais I, Li $H$, Sivasithamparam K, Barbetti MJ. Stabilization of resistance to Leptosphaeria maculans in Brassica napus-B. juncea recombinant lines and its introgression into spring-type Brassica napus. Plant Dis. 2008;92(8):1208-14.

58. Zou J, Hu D, Liu P, Raman H, Liu Z, Liu X, Parkin IAP, Chalhoub B, Meng J. Co-linearity and divergence of the A subgenome of Brassica juncea compared with other Brassica species carrying different A subgenomes. BMC Genomics. 2016;17(1):1-14.

59. Berrabah F, Bourcy M, Eschstruth A, Cayrel A, Guefrachi I, Mergaert P, Wen J, Jean V, Mysore KS, Gourion B, et al. A nonRD receptor-like kinase prevents nodule early senescence and defense-like reactions during symbiosis. New Phytol. 2014;203(4):1305-14.

60. Yeh Y-H, Chang Y-H, Huang P-Y, Huang J-B, Zimmerli L. Enhanced Arabidopsis pattern-triggered immunity by overexpression of cysteine-rich receptor-like kinases. Frontiers in Plant Science. 2015;6:322.

61. Gómez-Gómez L, Boller T. FLS2: An LRR receptor-like kinase involved in the perception of the bacterial elicitor flagellin in Arabidopsis. Mol Cell. 2000;5(6):1003-11.

62. Lloyd SR, Schoonbeek HJ, Trick M, Zipfel C, Ridout CJ. Methods to study PAMP-Triggered immunity in Brassica species. Mol Plant Microbe Interact. 2014;27(3):286-95.

63. Shinya T, Nakagawa T, Kaku H, Shibuya N. Chitin-mediated plant-fungal interactions: Catching, hiding and handshaking. Curr Opin Plant Biol. 2015:26:64-71.

64. Bourdais G, Burdiak Pł, Gauthier A, Nitsch L, Salojärvi J, Rayapuram C, Idänheimo N, Hunter K, Kimura S, Merilo E, et al. Large-Scale Phenomics Identifies Primary and Fine-Tuning Roles for CRKs in Responses Related to Oxidative Stress. PLoS Genet. 2015;11(7):e1005373.

65. Raman H, Dalton-Morgan J, Diffey S, Raman R, Alamery S, Edwards D, Batley J. SNP markers-based map construction and genome-wide linkage analysis in Brassica napus. Plant Biotechnol J. 2014;12(7):851-60.

66. Dilmaghani A, Balesdent MH, Didier JP, Wu C, Davey J, Barbetti MJ, Li H, Moreno-Rico O, Phillips D, Despeghel JP, et al. The Leptosphaeria maculans - Leptosphaeria biglobosa species complex in the American continent. Plant Pathol. 2009;58(6):1044-58.

67. Ma L, Borhan MH. The receptor-like kinase SOBIR1 interacts with Brassica napus LepR3 and is required for Leptosphaeria maculans AvrLm1-triggered immunity. Frontiers in Plant Science. 2015;6:933.

68. Ferrie AMR, Epp DJ, Keller WA. Evaluation of Brassica rapa L. genotypes for microspore culture response and identification of a highly embryogenic line. Plant Cell Rep. 1995;14(9):580-4.

69. R: A language and environment for statistical computing [http://www.Rproject.org/]

70. Ferreira ME, Rimmer SR, Williams PH, Osborn TC. Mapping loci controlling Brassica napus resistance to Leptosphaeria maculans under different screening conditions. Phytopathology. 1995;85(2):213-7.
71. Utermark J, Karlovsky P: Genetic transformation of filamentous fungi by Agrobacterium tumefaciens. In: Nature Protocol Exchange. Nature Publishing Group; 2008. http://www.nature.com/protocolexchange/protocols/427

72. Parlange F, Daverdin G, Fudal I, Kuhn M-L, Balesdent M-H, Blaise F, GrezesBesset B, Rouxel T. Leptosphaeria maculans avirulence gene AvrLm4-7 confers a dual recognition specificity by the $R / m 4$ and $R / m 7$ resistance genes of oilseed rape, and circumvents R/m4-mediated recognition through a single amino acid change. Mol Microbiol. 2009;71(4):851-63.

73. Larkan NJ, Lydiate DJ, Yu F, Rimmer SR, Borhan MH. Co-localisation of the blackleg resistance genes R/m2 and LepR3 on Brassica napus chromosome A10. BMC Plant Biol. 2014;14(1):1595.

74. Raman H, Raman R, Kilian A, Detering F, Long Y, Edwards D, Parkin IAP, Sharpe AG, Nelson MN, Larkan N, et al. A consensus map of rapeseed (Brassica napus L.) based on diversity array technology markers: Applications in genetic dissection of qualitative and quantitative traits. BMC Genomics. 2013;14(1):277.

75. Li H, Ye G, Wang J. A modified algorithm for the improvement of composite interval mapping. Genetics. 2007;175(1):361-74.

76. Li H, Ribaut JM, Li Z, Wang J. Inclusive composite interval mapping (ICIM) for digenic epistasis of quantitative traits in biparental populations. Theor Appl Genet. 2008;116(2):243-60.

77. Zhang L, Li H, Wang J. The statistical power of Inclusive Composite Interval Mapping in detecting digenic epistasis showing common $\mathrm{F}_{2}$ segregation ratios. J Integr Plant Biol. 2012;54(4):270-9.

78. Voorrips RE. Mapchart: Software for the graphical presentation of linkage maps and QTLs. Journal Hered. 2002;93(1):77-8.

\section{Submit your next manuscript to BioMed Central and we will help you at every step:}

- We accept pre-submission inquiries

- Our selector tool helps you to find the most relevant journal

- We provide round the clock customer support

- Convenient online submission

- Thorough peer review

- Inclusion in PubMed and all major indexing services

- Maximum visibility for your research

Submit your manuscript at www.biomedcentral.com/submit
Biomed Central 